\title{
Ultrafast Charge Rearrangement and Nuclear Dynamics upon Inner-Shell Multiple Ionization of Small Polyatomic Molecules
}

\author{
B. Erk, ${ }^{1,2}$ D. Rolles, ${ }^{1,3,4}$ L. Foucar, ${ }^{1,3}$ B. Rudek, ${ }^{1,2,4}$ S. W. Epp, ${ }^{1,2}$ M. Cryle, ${ }^{3}$ C. Bostedt, ${ }^{5}$ S. Schorb, ${ }^{5,6}$ J. Bozek, ${ }^{5}$ \\ A. Rouzee, ${ }^{7}$ A. Hundertmark, ${ }^{7}$ T. Marchenko, ${ }^{8}$ M. Simon, ${ }^{8}$ F. Filsinger, ${ }^{9}$ L. Christensen, ${ }^{10}$ S. De, ${ }^{11,12}$ S. Trippel, ${ }^{13}$ \\ J. Küpper, ${ }^{13,14}$ H. Stapelfeldt, ${ }^{11}$ S. Wada, ${ }^{5,15}$ K. Ueda, ${ }^{16}$ M. Swiggers, ${ }^{5}$ M. Messerschmidt, ${ }^{5}$ C. D. Schröter, ${ }^{2}$ \\ R. Moshammer, ${ }^{2}$ I. Schlichting, ${ }^{1,3}$ J. Ullrich, $^{17,1,2}$ and A. Rudenko ${ }^{18,1,2}$ \\ ${ }^{1}$ Max Planck Advanced Study Group (ASG) at CFEL, 22761 Hamburg, Germany \\ ${ }^{2}$ Max Planck Institut für Kernphysik, 69117 Heidelberg, Germany \\ ${ }^{3}$ Max Planck Institut für medizinische Forschung, 69120 Heidelberg, Germany \\ ${ }^{4}$ DESY, 22607 Hamburg, Germany \\ ${ }^{5}$ Linac Coherent Light Source, SLAC National Accelerator Laboratory, Menlo Park, California 94025, USA \\ ${ }^{6}$ Institut für Optik und Atomare Physik, Technische Universität Berlin, 10623 Berlin, Germany \\ ${ }^{7}$ Max Born Institute, 12489 Berlin, Germany \\ ${ }^{8}$ Laboratoire de Chimie Physique-Matière et Rayonnement, CNRS and UPMC, 75231 Paris, France \\ ${ }^{9}$ Fritz-Haber-Institut der Max-Planck-Gesellschaft, 14195 Berlin, Germany \\ ${ }^{10}$ Department of Physics and Astronomy, Aarhus University, 8000 Aarhus C, Denmark \\ ${ }^{11}$ Department of Chemistry, Aarhus University, 8000 Aarhus C, Denmark \\ ${ }^{12}$ Saha Institute of Nuclear Physics, 700064 Kolkata, India \\ ${ }^{13}$ Center for Free-Electron Laser Science (CFEL), DESY, 22607 Hamburg, Germany \\ ${ }^{14}$ Department of Physics, University of Hamburg, 22761 Hamburg, Germany \\ ${ }^{15}$ Department of Physical Science, Hiroshima University, 739-8526 Higashi-Hiroshima, Japan \\ ${ }^{16}$ IMRAM, Tohoku University, 980-8577 Sendai, Japan \\ ${ }^{17}$ Physikalisch-Technische Bundesanstalt, 38116 Braunschweig, Germany \\ ${ }^{18}$ J. R. Macdonald Laboratory, Department of Physics, Kansas State University, Manhattan, Kansas 66506, USA
}

(Received 2 October 2012; published 30 January 2013)

\begin{abstract}
Ionization and fragmentation of methylselenol $\left(\mathrm{CH}_{3} \mathrm{SeH}\right)$ molecules by intense $\left(>10^{17} \mathrm{~W} / \mathrm{cm}^{2}\right) 5 \mathrm{fs}$ $\mathrm{x}$-ray pulses $(\hbar \omega=2 \mathrm{keV})$ are studied by coincident ion momentum spectroscopy. We contrast the measured charge state distribution with data on atomic $\mathrm{Kr}$, determine kinetic energies of resulting ionic fragments, and compare them to the outcome of a Coulomb explosion model. We find signatures of ultrafast charge redistribution from the inner-shell ionized Se atom to its molecular partners, and observe significant displacement of the atomic constituents in the course of multiple ionization.
\end{abstract}

DOI: 10.1103/PhysRevLett.110.053003

PACS numbers: 33.80.Eh, 33.80.Rv, 33.90.+h

The microscopic response of a polyatomic system to inner-shell photoabsorption, i.e., the electronic relaxation dynamics and motion of atomic nuclei after creation of one or several core-shell vacancies, plays a crucial role for our understanding of x-ray interactions with matter. It is also essential for most applications of novel ultraintense, ultrafast x-ray sources such as x-ray free-electron lasers (XFELs), in particular, for coherent imaging techniques which rely on the feasibility of the ground-breaking "diffract-before-destroy" concept [1]. For a given object this critically depends on the mechanisms and time scales of both electronic and nuclear rearrangement, which defines local radiation damage to the sample irradiated by an intense $\mathrm{x}$-ray pulse.

The availability of the first hard x-ray FEL, the Linac Coherent Light Source (LCLS) [2], enabled proof-ofprinciple imaging experiments on single nanoscale particles [3-6] and nanocrystals [7-9], and triggered dedicated radiation damage studies [8,9]. In parallel, measurements aimed at understanding the behavior of simple systems such as isolated atoms [10-12], diatomic molecules [13-15], and small rare gas clusters [16] were conducted. These experiments revealed novel effects such as double or multiple core-hole creation and frustrated x-ray absorption [10,13-16], and showed that very high levels of ionization can be achieved (e.g., up to $36+$ in xenon atoms) [12].

In this Letter, we present the first experimental results on the ionization and fragmentation dynamics of a polyatomic molecule, methylselenol $\left[\mathrm{CH}_{3} \mathrm{SeH}\right.$, Fig. 1(a)], irradiated by intense $\left(>10^{17} \mathrm{~W} / \mathrm{cm}^{2}\right)$ ultrashort $\mathrm{x}$-ray pulses at $2 \mathrm{keV}$ photon energy, and on the extent of radiation damage induced in such a system. Methylselenol represents an ideal target for studying the microscopic response of a small polyatomic system since it contains one high- $Z$ atom (Se), which has a photoabsorption cross section much higher than the other atoms in the molecule $(0.4 \mathrm{Mb}$ compared to $\sim 0.006 \mathrm{Mb}$ ), such that the initial photoabsorption almost exclusively occurs at the Se $L$ edge, and, therefore, is well localized. Comparing the ionization of the molecule to the case of an isolated $\mathrm{Kr}$ atom, which has a similar 
(a)

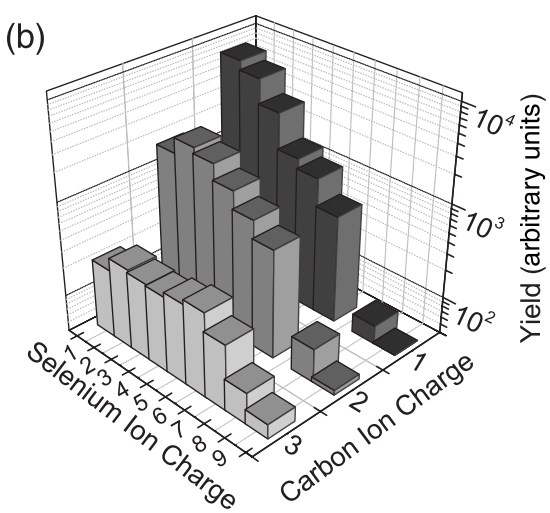

(c)

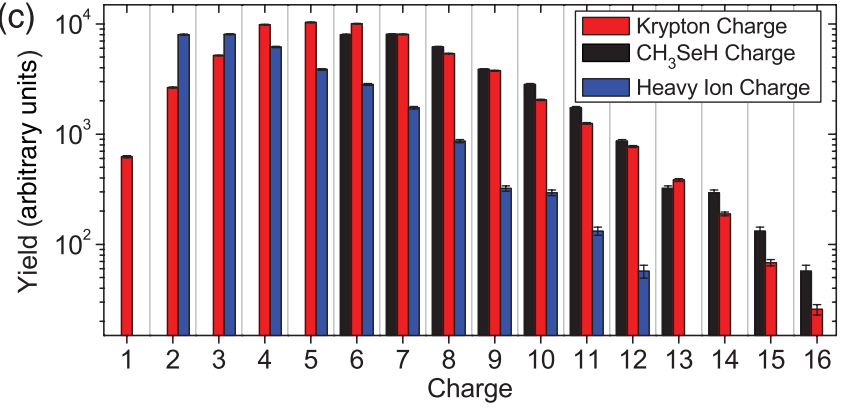

FIG. 1 (color). (a) Geometry of the methylselenol molecule. (b) Measured yield of coincident $\mathrm{Se}$ and $\mathrm{C}$ ion pairs for different final charge states. (c) Sum charge induced on the molecule compared to the charge state distribution observed in $\mathrm{Kr}$ under the same conditions. The heavy ion charge represents the sum of $\mathrm{Se}$ and $\mathrm{C}$ charges measured in coincidence, whereas the $\mathrm{CH}_{3} \mathrm{SeH}$ charge denotes the total charge of the molecule assuming that four $\mathrm{H}^{+}$ions were produced.

electronic configuration and absorption cross section $(0.5 \mathrm{Mb})$, we find that while the total charge induced on the system (up to 16 electrons are removed) is the same in both cases, the highest final charge state of Se is considerably lower $\left(\mathrm{Se}^{9+}\right.$ as opposed to $\left.\mathrm{Kr}^{16+}\right)$. By measuring kinetic energy distribution (KED) of ionic fragments for a given final charge state, and comparing them to the outcome of a simple Coulomb explosion (CE) model, we trace the evolution of the molecular geometry both during and after the x-ray pulse and observe considerable displacement of the nuclei on the time scale of sequential multiple ionization and Auger decay. We find surprisingly high fragment energies for channels where the measured charge of the $\mathrm{C}$ ions is higher than that of Se, pointing towards ultrafast charge rearrangement occurring en route to these final states.

The experiment was performed at the LCLS AMO beam line at SLAC using the CFEL ASG Multi-Purpose end station (CAMP) [17]. The LCLS x-ray pulses at $2 \mathrm{keV}$ photon energy (peak fluence on target $\sim 15 \mu \mathrm{J} / \mu \mathrm{m}^{2}$, nominal pulse duration $\sim 5$ fs FWHM [18]) were focused to a $\sim 10 \mu \mathrm{m}^{2}$ spot onto a supersonic jet of $\mathrm{Kr}$ atoms or methylselenol molecules. The produced ions were projected onto a time- and position-sensitive microchannel-plate detector with a delay-line anode, allowing for reconstruction of the 3D momentum vectors of multiple fragments in coincidence.
Upon inner-shell ionization at the Se $L$ edge, the methylselenol molecule breaks up into few ionic fragments. Figure 1(b) depicts the measured charge state distribution of Se and $\mathrm{C}$ ions detected in coincidence. While the yield typically decreases with increasing total charge, for a given sum of Se and C charge the channels where the $\mathrm{C}$ fragment is charged higher than Se have lower abundance, since photons are almost exclusively absorbed by the Se constituent.

Considering only selenium and carbon ions, the highest charge state combination observed with statistical significance is $\mathrm{Se}^{9+}+\mathrm{C}^{3+}$. Although proton fragments were detected as well, we do not use them for the determination of the total charge state of the molecule, because typically more than one proton is ejected, and the total detection efficiency becomes very low for coincidences of more than three particles. Therefore, in order to estimate the total charge, we assume that all hydrogen fragments are charged, which is well justified for higher charge states [19], but might deviate considerably for lower ones. The deduced total charge for the methylselenol molecule is shown in Fig. 1(c) in comparison with the charge state distribution measured for $\mathrm{Kr}$ atom under identical experimental conditions.

The results in Fig. 1(c) show that although the individual charge of the $\mathrm{Se}$ atom is much lower than for $\mathrm{Kr}$, the maximum total charge induced on the molecule $(16+)$ and the overall charge state distribution is very similar to the atomic case. Since the highest charge state produced by one-photon ionization at this photon energy is $\mathrm{Kr}^{7+}$ [20], a significant part of the distribution results from multiphoton absorption. This is illustrated in Fig. 2, where the measured yields of several representative coincident channels are plotted as a function of the LCLS pulse energy.

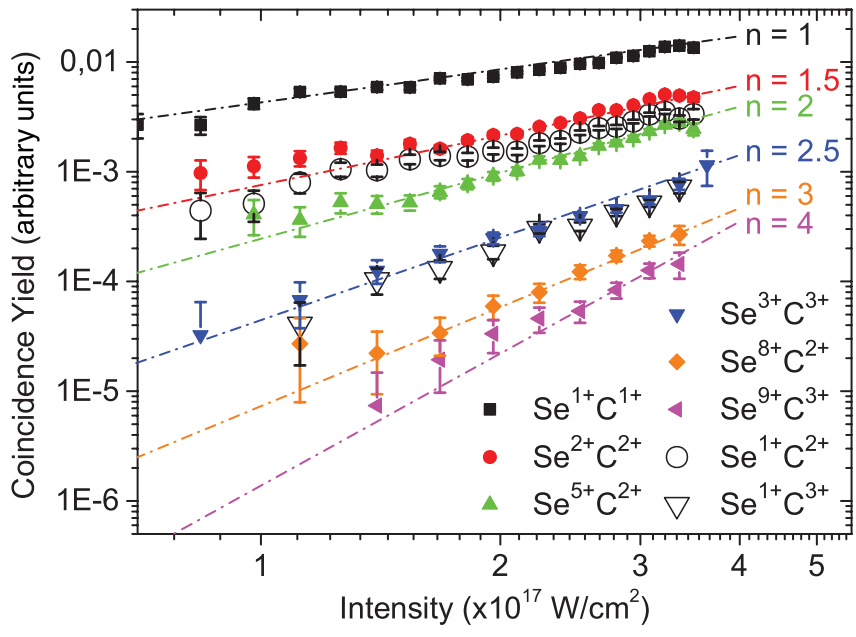

FIG. 2 (color). Yields of 8 exemplary heavy-ion coincident channels measured as a function of the FEL intensity. The power dependence of the ion yield as deduced from the slope in this double logarithmic representation gives insight into the number of photons $n$ needed to reach a certain final charge state (see text). To guide the eye, lines with slopes indicated on the right are drawn through the experimental data. 
Since according to perturbation theory, the ionization yield $Y$ should increase with the light intensity as $Y \sim \sigma_{n} I^{n}$, where $I$ is the light intensity (proportional to the pulse energy), $\sigma_{n}$ the generalized n-photon cross section, and $n$ the number of involved photons [21] for a certain pathway, which can be determined from the power dependence of the corresponding yield curve. The measured slopes indicate that while the lowest charge state combinations are mainly produced by one photon processes, all final states with the sum charge of the two heavy fragments $q_{\mathrm{C}}+$ $q_{\mathrm{Se}}>4$, as well as all pathways involving doubly or triply charged carbon ions are predominantly reached by the absorption of 2 or 3 photons (up to 4 for the highest charge states observed). Noninteger slopes could result from a superposition of pathways involving different number of absorbed photons at a given intensity. Moreover, the uncertainty in the number of created protons mentioned above might result in an averaging over several total-charge states of the molecule for a given sum of the Se and C charges. This could also lead to noninteger $n$ in Fig. 2. It should also be noted that several channels exhibit a pronounced change of the slope indicating an intensity-dependent transition between $n$ - and $(n+1)$-photon processes.

The dominant ionization mechanism in the x-ray regime is core-shell photoionization followed by Auger decay (except for $K$-shell ionization of heavy atoms, where fluorescent decay dominates). For $\mathrm{Se}$ or $\mathrm{Kr}$ a vacancy in the $L$ shell causes multiple ionization via an Auger cascade. The first relaxation step with the highest probability is an "intra-atomic" LMM Auger decay (hole lifetime $\sim 500$ as) in the Se atom producing mainly $3 d$ (less likely $3 p$ ) vacancies [22]. It is followed by a decay of these $M$-shell vacancies involving the valence electrons of $\mathrm{Se}$ and its neighbors (MVV Auger), which occurs, on a $\sim 10 \mathrm{fs}$ time scale [22]. The latter step causes a considerable portion of the total charge to be distributed over all molecular constituents even though the initial innershell photoabsorption and the first Auger decay are very likely to be localized on the Se atom.

To obtain insight into the charge rearrangement and nuclear dynamics, we examine the measured kinetic energies of the ionic fragments shown in Figs. 3 and 4. In Figs. 3(a)-3(c), the measured KEDs of selenium ions (a), carbon ions (b), and protons (c) detected in coincidence are presented for four different charge state combinations. In all cases, we observe a broad energy distribution with its maximum clearly shifting towards higher energies with increasing total charge state. In order to relate these results to the molecular geometry, we performed a simple simulation of the molecular Coulomb explosion, assuming instantaneous removal of the electrons at the equilibrium bond lengths and angles. The results of the simulation are shown as vertical lines in Figs. 3(a)-3(c).

For the lowest charge state $\left(\mathrm{Se}^{+}+\mathrm{C}^{+}\right)$, the simulated energies of carbon and selenium ions fit well to the
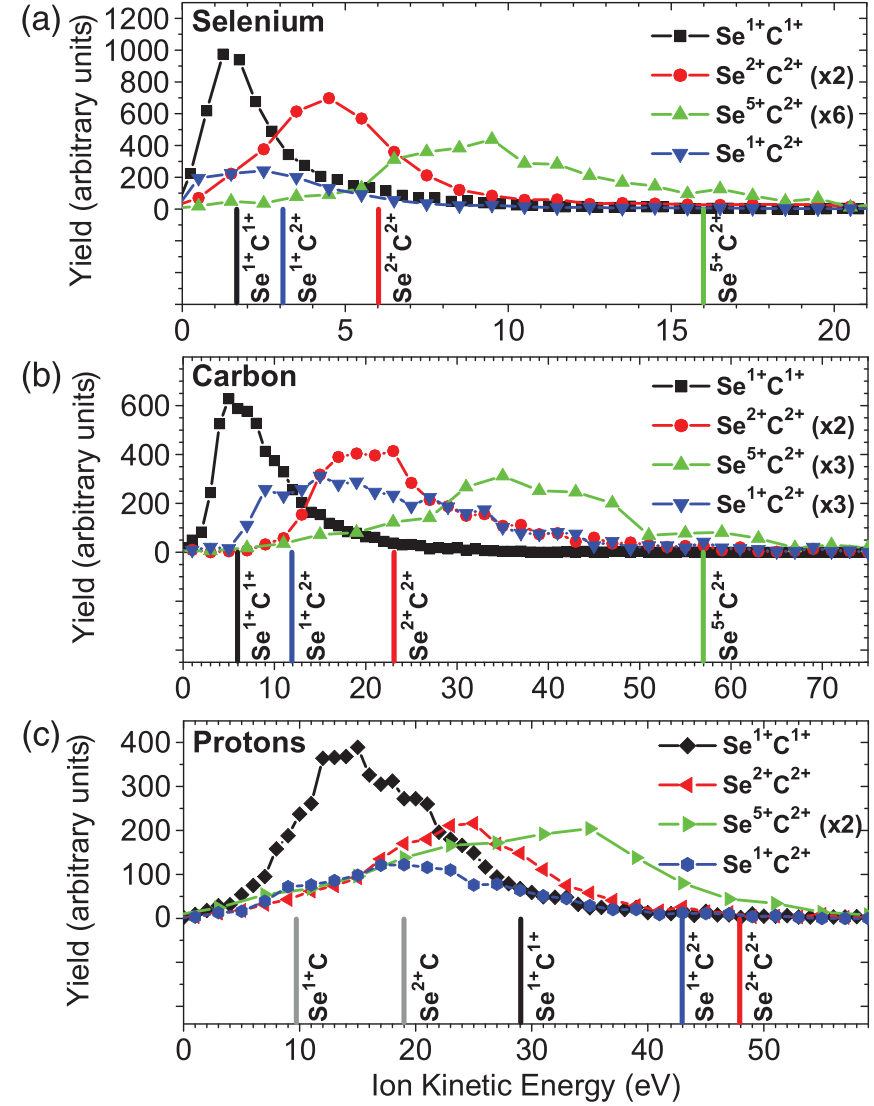

FIG. 3 (color). The kinetic energy distributions of $\mathrm{Se}, \mathrm{C}$, and $\mathrm{H}$ ions for four different fragmentation channels. Vertical lines of the same color indicate simulated energies for given charge states assuming the equilibrium geometry of the neutral molecule (see text).

maximum of the KED, whereas they increasingly overestimate the experimental results for the higher ones (the $\mathrm{Se}^{+}+\mathrm{C}^{2+}$ channel behaves differently and will be discussed below). The simulated proton energies are much larger than the measured ones for all charge states. The considerable deviation of the measured KEDs from the simulated CE energies for higher charge states is a direct measure of the motion of the nuclei on the time scale of multiple ionization. Low charge states are predominantly produced by single photon absorption, such that fast dissociation starts upon ultrafast core-vacancy decay resulting in kinetic energies of the heavy ions that are in reasonable agreement with the simulations for equilibrium geometry. This is in line with the outcome of earlier synchrotron experiment for the case of $\mathrm{S}(1 s)$ ionization of OCS molecules [23]. In contrast, the lightest ionic fragmentsprotons-are considerably displaced even within this very short time (in $1 \mathrm{fs}$ a proton with one atomic unit of energy $(27.2 \mathrm{eV})$ moves about $3.5 \AA$ A, i.e., more than a bond length per fs). Therefore, the measured protons never exhibit the simulated $\mathrm{CE}$ energies corresponding to the equilibrium internuclear distances $R_{\text {eq }}$ [Fig. 3(c)]. 


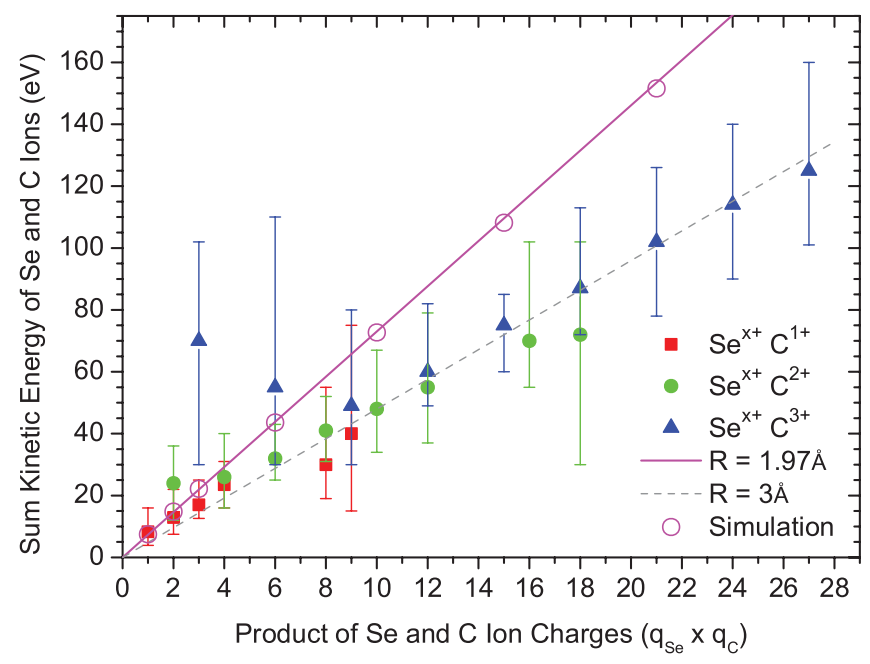

FIG. 4 (color). Measured (full symbols) and calculated (lines) sum kinetic energy of $\mathrm{Se}$ and $\mathrm{C}$ ions as a function of the product of their charge states. Open circles represent the results of the $\mathrm{CE}$ simulation assuming that all four $\mathrm{H}$ atoms are charged. The solid line depicts the total energy of Se and $\mathrm{C}$ ions for $\mathrm{CE}$ at the equilibrium $R$, whereas the dashed line corresponds $R=3 \AA$ (see text). Note that for the experimental data the symbols represent the maximum of the sum energy distribution, while vertical bars indicate the nonsymmetrical full width at half maximum, and not the error bars.

In general, the production of the higher charge states involves sequential absorption of two or more photons (with a time delay on a femtosecond scale in-between the absorption steps), each triggering new Auger cascades. However, the removal of the first few electrons typically already triggers the dissociation of the molecule [24]. By the time the final charge state is reached, the bond lengths have significantly increased, leading to lower Coulomb repulsion energies for a given charge state combination [25], and, thus, larger deviations from the CE model (as can be clearly observed in Fig. 3).

The dynamics of the heavy nuclei on the time scale of multiple ionization is illustrated in Fig. 4, where the measured and calculated sum energy of Se and C ions is plotted as a function of the product of their charge states. Note that for the experimental data the position of the maximum of the sum KED is given by the symbol while the nonsymmetrical full width at half maximum of the distribution is indicated by the vertical bars. In this representation, the assumption of an instantaneous CE of a two-center system at a certain time results in a straight line with the slope given by the inverse of the internuclear distance $R$. While the measured sum energies for the two lowest charge state combinations, $\mathrm{Se}^{+}+\mathrm{C}^{+}$and $\mathrm{Se}^{2+}+\mathrm{C}^{+}$, fit the line corresponding to the equilibrium internuclear distance $\left(R_{\text {eq }}=1.97 \AA\right)$, the experimental data for the higher ones show considerably lower energies. These values can be reproduced by the line given by $R=3 \AA$. Thus, the latter value provides an estimate for the lower limit of molecular deformation during few-photon absorption and subsequent Auger decay; i.e., the molecule is stretched by $\sim 50 \%$ of $R_{\text {eq }}$ within 5 to 10 fs.

Although most of the measured fragment energies in Fig. 4 are significantly lower than the $\mathrm{CE}$ model values predicted for $R_{\mathrm{eq}}$, the channels for which $q_{\mathrm{C}}>q_{\mathrm{Se}}$ exhibit clearly different behavior. Here, the measured KEDs for heavy ions are very broad, as can be seen for the $\mathrm{Se}^{+}+\mathrm{C}^{2+}$ channel in Figs. 3(a) and 3(b), and the maxima of the measured sum energy distributions lie considerably higher than the simulated CE values. It is especially pronounced for the $\mathrm{Se}^{+}+\mathrm{C}^{3+}$ channel, for which the measured energy is similar to the value simulated for much higher final states, such as $\mathrm{Se}^{3+}+\mathrm{C}^{3+}$ or $\mathrm{Se}^{5+}+\mathrm{C}^{2+}$. We would like to point out that this observation is very surprising since the assumption of the instantaneous $\mathrm{CE}$ at $R_{\mathrm{eq}}$ normally provides the upper limit for the ion kinetic energies detected upon a breakup of a multiply ionized molecule. The most plausible explanation implies that the Se-C complex is initially (at small $R$ ) charged higher than in the measured final state, resulting in early CE with high fragment energies, and, subsequently, the charge is shared with the neighboring protons. This hypothesis is supported by the fact that not only the fragment sum energies in Fig. 4, but also the intensity dependence (Fig. 2) and the total yield [Fig. 1(b)] of the $\mathrm{Se}^{+}+\mathrm{C}^{3+}$ channel closely resembles those of $\mathrm{Se}^{3+}+\mathrm{C}^{3+}$ or even higher channels. The measured sum energy for $\mathrm{Se}^{+}+\mathrm{C}^{3+}$ in Fig. 4 is similar to the experimental value for $\mathrm{Se}^{5+}+\mathrm{C}^{3+}$, the intermediate state which differs from the final state by the total number of the protons available. Similar charge rearrangement might also be responsible for the high-energy tails of the KEDs in Fig. 3, extending beyond the CE simulations for $R_{\text {eq }}$.

According to the measured intensity-dependent yields, the final states which $q_{C}>q_{S e}$ typically require the absorption of more than one photon (see $\mathrm{Se}^{+}+\mathrm{C}^{2+}$ and $\mathrm{Se}^{+}+$ $\mathrm{C}^{3+}$ channels in Fig. 2). Although one of the photons might be absorbed by the carbon $K$ shell, two-photon absorption by the carbon atom is extremely unlikely due to the low cross section. Therefore, the pathways to these final states most likely involve interatomic molecular Auger decay, "filling" Se inner-shell vacancies with valence electrons of the $\mathrm{C}$ atom [25]. This, as well as charging of the hydrogen fragments mentioned above, is more probable to happen at small internuclear distances [26]. Thus, the presence of these asymmetric channels along with their anomalously high CE energies most likely reflects the ultrafast charge rearrangement involving inner-shell vacancies and occurring before any significant nuclear motion. An alternative explanation would imply a charge redistribution mechanism through valence orbitals proceeding on the same time scale.

In conclusion, we have studied multiple ionization and fragmentation of a small organic molecule with an 
embedded heavy atom upon absorption of up to four x-ray photons. We show that the molecular geometry, including the $\mathrm{Se}-\mathrm{C}$ bond length, changes considerably already during the ionization process ( $>1 \AA$ displacement for the heavy fragments and considerably more for the protons). We observe signatures of ultrafast charge redistribution from the absorbing atom to the molecular environment that results in a lower charge of the heavy atom embedded in the molecule as compared to an isolated atom, as well as in anomalously high fragment energies for the final states where carbon is charged higher than the absorbing selenium atom. The subtleties of such charge redistribution processes can be efficiently studied in a pump-probe experiment, where the molecule is predissociated, e.g., by a femtosecond optical laser, and subsequent x-ray fragmentation is examined for different internuclear distances between the fragments.

Our results have direct implications for coherent diffractive imaging of single molecules, pointing to the creation of local "hot spots" of increased radiation damage in the vicinity of heavy atoms (e.g., iron, phosphorus, or sulfur atoms contained in the biological molecules) [9], and indicating that all imaging schemes employing intense $\mathrm{x}$-ray pulses should take into account considerable motion of the atomic constituents on time scales as short as a few femtoseconds.

Parts of this research were carried out at the Linac Coherent Light Source (LCLS) at the SLAC National Accelerator Laboratory. LCLS is an Office of Science User Facility operated for the U.S. Department of Energy Office of Science by Stanford University. We acknowledge the Max Planck Society for funding the development and operation of the CAMP instrument within the ASG at CFEL. A. R. acknowledges the support from Chemical Sciences, Geosciences, and Biosciences Division, Office of Basic Energy Sciences, Office of Science, US Department of Energy. H. S., L. C., and S. D. acknowledge support from the Carlsberg Foundation. We are grateful to C. Schmidt for technical support, to J. M. Rost for valuable discussions, and to the SLAC staff for their support and hospitality during the beam time.
[1] R. Neutze, R. Wouts, D. van der Spoel, E. Weckert, and J. Hajdu, Nature (London) 406, 752 (2000).

[2] P. Emma et al., Nat. Photonics 4, 641 (2010).

[3] M. M. Seibert et al., Nature (London) 470, 78 (2011).

[4] T. Gorkhover et al., Phys. Rev. Lett. 108, 245005 (2012).

[5] D. Loh et al., Nature (London) 486, 513 (2012).

[6] S. Kassemeyer et al., Opt. Express 20, 4149 (2012).

[7] H.N. Chapman et al., Nature (London) 470, 73 (2011).

[8] A. Barty et al., Nat. Photonics 6, 35 (2012).

[9] L. Lomb et al., Phys. Rev. B 84, 214111 (2011).

[10] L. Young et al., Nature (London) 466, 56 (2010).

[11] G. Doumy et al., Phys. Rev. Lett. 106, 083002 (2011).

[12] B. Rudek et al., Nat. Photonics 6, 858 (2012).

[13] M. Hoener et al., Phys. Rev. Lett. 104, 253002 (2010).

[14] J.P. Cryan et al., Phys. Rev. Lett. 105, 083004 (2010).

[15] N. Berrah et al., Proc. Natl. Acad. Sci. U.S.A. 108, 16912 (2011).

[16] S. Schorb et al., Phys. Rev. Lett. 108, 233401 (2012).

[17] L. Strüder et al., Nucl. Instrum. Methods Phys. Res., Sect. A 614, 483 (2010).

[18] For a discussion on the real LCLS pulse duration in the low bunch charge mode, see Y. Ding et al., Phys. Rev. Lett. 102, 254801 (2009).

[19] E. Shigemasa, K. Ueda, Y. Sato, A. Yagishita, H. Maezawa, T. Sasaki, M. Ukai, and T. Hayaishi, Phys. Scr. 41, 67 (1990).

[20] Y. Morishita, Y. Tamenori, K. Okada, T. Oyama, K. Yamamoto, K. Tabayashi, T. Ibuki, K. Moribayashi, and I. H. Suzuki, J. Phys. B 39, 1323 (2006).

[21] N. B. Delone and V. P. Krainov, Multiphoton Processes in Atoms (Springer-Verlag, Berlin, 2000).

[22] J. L. Campbell and T. Papp, At. Data Nucl. Data Tables 77, 1 (2001).

[23] U. Ankerhold, B. Esser, and F. von Busch, J. Phys. B 30, 1207 (1997).

[24] M. Simon, M. Lavollée, M. Meyer, and P. Morin, J. Electron Spectrosc. Relat. Phenom. 79, 401 (1996).

[25] R. Dunford et al., Phys. Rev. A 86, 033401 (2012).

[26] Th. A. Carlson and R. M. White, J. Chem. Phys. 44, 4510 (1966). 